\title{
EVOLVE: a tool for evaluating the design of older people's housing
}

\author{
Alan Lewis \\ Research Associate, School of Architecture, University of Sheffield, UK \\ Judith Torrington', Sarah Barnes2 , Robin Darton ${ }^{3}$, Jacquetta Holder ${ }^{3}$, Kevin McKee ${ }^{4}$, \\ Ann Netten ${ }^{3}$, Alison Orrell ${ }^{5}$ \\ ${ }^{1}$ School of Architecture, University of Sheffield, UK \\ 2 School of Health and Related Research, University of Sheffield, UK \\ ${ }^{3}$ PSSRU, School of Social Policy, Sociology and Social Research, University of Kent, UK \\ ${ }^{4}$ School of Health and Social Studies, Dalarna University, and Dalarna Research Institute, Sweden \\ ${ }^{5}$ Sheffield Institute for Studies on Ageing, University of Sheffield, UK
}

\begin{abstract}
EVOLVE is a tool for evaluating the design of housing for older people. It is used to assess how well a building contributes to the physical support and personal well-being of older people. Developed from research into extra care housing, it can be used for a variety of building types, including sheltered housing and individual private houses. The tool can be used by architects, housing providers, commissioners, researchers and individual tenants or home owners. EVOLVE can be used as a briefing document or an aid to design. It can provide a rational basis to the selection of proposals in a competitive procurement process. The EVOLVE tool can also be used to evaluate existing housing stock, including schemes where remodelling is under consideration.
\end{abstract}

\section{Key words}

building evaluation tool; housing; older people; ageing

\section{Introduction}

The detrimental effects of ageing are welldocumented. Poor physical and mental health, physical or sensory impairments, social isolation and comparative poverty are common difficulties for older people. Tackling these issues is a major concern in an ageing society.
Housing design can help to ameliorate many of these problems. Well-designed housing can improve people's quality of life (Parker et al, 2004; Evans et al, 2002). It can enable older people to retain their independence for longer (Wahl et al, 2009), preventing or delaying the need for institutional 
care and reducing care costs (CLG, 2008 pp36-7; Thomson et al, 2001).

To facilitate evaluation of the design of housing for older people, a tool has been created by researchers from the University of Sheffield, in collaboration with PSSRU (Personal Social Services Research Unit), University of Kent, and supported by the Housing LIN (Learning and Improvement Network) and EAC (Elderly Accommodation Counsel) and funded by the EPSRC (Engineering and Physical Sciences Research Council).

Known as EVOLVE (Evaluation of Older People's Living Environments), the tool offers a way of evaluating the design of housing schemes or individual dwellings to assess their suitability as accommodation for older people.

EVOLVE is essentially a set of checklists covering all those areas of a housing scheme used by residents, including apartments and bungalows, communal facilities, circulation spaces (for example corridors, lifts and stairs), staff facilities, scheme site and location.

Although the EVOLVE tool was developed from research into extra care housing, the tool can be used for a range of housing types, including retirement villages, sheltered housing and individual private houses, but excluding residential care homes and nursing homes, for which a separate tool has been developed: SCEAM (Sheffield Care Environment Assessment Matrix). EVOLVE can be used at all stages in the development cycle, from design to postoccupancy evaluation.

The tool complements existing standards and codes, such as Lifetime Homes standards and BREEAM (BRE Environmental Assessment Method), by focusing specifically on the housing needs of older people and by featuring a high level of detail.

The tool can be used by architects, housing providers, commissioners and researchers, or by individuals who are considering moving into a new home.

The EVOLVE tool can be used:

- at the design stage

- as an aid to architects and providers, enabling the design and commissioning process to be underpinned by a firm evidence base

- as an aid to commissioners, providing a rational basis for evaluation and selection of alternative proposals in competitive procurement processes

- for the evaluation of existing buildings

- as an aid to housing managers in long-term evaluation and upgrading of existing estates

- as an aid to housing providers in exploring the feasibility of remodelling existing buildings

- in academic research, enabling aspects of the built environment to be evaluated against other variables, such as quality of life measures.

Electronic and paper versions of the tool are available. Walk-through evaluations can be undertaken using a small computer such as a netbook or the paper-based version of the tool. The electronic version of the tool can also be used in an office, at the design stage or as a way of scoring an evaluation made using the paperbased version of the tool.

\section{Tool development}

The items in the EVOLVE tool were generated from a number of sources, including:

- a review of the existing academic literature

- a review of existing standards and guidance on housing design for older people

- a review of recent examples of older people's housing

- focus groups with people living in four extra care schemes in England of varying typology, specifically:

- a small new-build scheme (not for profit)

- a small new-build scheme (private sector)

- a remodelled scheme

- a retirement village

- a focus group with relatives of people living in an extra care scheme

- interviews with architects specialising in the design of housing for older people, commissioners, housing providers, extra care scheme managers and staff.

\section{Testing of the EVOLVE tool}

Draft versions of the tool were piloted in five extra care schemes, and revisions were made to the tool after each exercise. The tool was then tested through surveys of 23 extra care schemes 
in England. The scores obtained support face validity, that is, there were a range of scores across the schemes with no apparent ceiling or flooring effect. The findings also support content validity, as scores reflected expectations. For example, retirement villages scored highly on support for socialising within the scheme, but scored less well on support for socialising outside the scheme, as might be expected given the wide range of facilities available in most villages and the tendency for villages to be located in rural or suburban areas. By contrast, small new-build schemes, which tend to have fewer facilities and are often located in urban or suburban areas close to amenities like shops, scored comparatively low on support for socialising within the scheme, but scored more highly on support for socialising outside the scheme.

The results of the survey suggest that the standard of design of new-build extra care housing is fairly high, but there is much room for improvement. Most schemes scored lower than expected for physical and sensory support, which is a concern, given the prevalence of physical and sensory impairments among older people. There is clearly scope for a tool such as EVOLVE to help improve design standards.

\section{Validation exercises}

During the survey, a series of inter-rater reliability tests were undertaken to see whether two researchers working independently achieved similar scores using the tool. These tests were undertaken in three schemes, and the schemelevel scores obtained by the two researchers had strong inter-rater reliability.

Following the conclusion of the survey, a series of validation exercises were undertaken to test the tool further and improve its usability. These exercises involved seven field workers using the tool to evaluate 12 extra care schemes. Fieldworkers were asked to comment on their experience of using the tool and to identify particular items that caused difficulties. These observations were used to revise the tool after each exercise, principally by re-wording items and adding a glossary to make the tool easier to use.
It was also established that it takes approximately one day to evaluate a small housing scheme, and one and a half days to survey a retirement village.

\section{Electronic version of EVOLVE}

From the paper-based version of the tool used in the survey of extra care schemes, an electronic version of EVOLVE was created using Microsoft Excel software. This simplifies the process because the summative scores which result from completing the tool are calculated by computer.

\section{Description of tool}

EVOLVE consists of a series of questions, or items, about the building under evaluation. There are 487 items for a single dwelling, and 2020 items for a housing scheme, assuming that one apartment or bungalow is assessed, although not all the items will be relevant in every case.

\section{Accommodation schedule}

Items are arranged in checklists, with a separate checklist for each room or space. The tool features an accommodation schedule for recording what rooms and facilities are present in the scheme under evaluation. Where particular rooms are absent from a scheme, the relevant checklists will drop out of the scoring. For example, if the apartment under evaluation has only one bedroom, the checklist for the second bedroom will appear greyed out and the maximum potential score will be reduced to take this into account. The accommodation schedule also displays the number of items in each checklist and indicates how many items have yet to be completed.

\section{Files in the EVOLVE toolkit folder}

In its electronic form, the EVOLVE toolkit folder contains a separate Microsoft Excel file for each area of the housing scheme under evaluation, including:

- living unit (for example apartment or bungalow)

- communal facilities

- circulation spaces (for example corridors, lifts and stairs)

- staff facilities

- scheme site and location. 


\section{Generic items}

Each file has a checklist for generic items. These are items that relate to features that are identical throughout, such as the width of doors and the design of radiators. By completing items in the generic checklist, relevant items in subsequent checklists will be completed automatically. This can save time in the evaluation of schemes with a lot of standardisation. See Table 1, below.

\section{Scoring}

In scoring each item in the EVOLVE Tool, there are four options.

- Yes - the statement is true; for example, the item 'The lounge has natural light' would be scored as 'yes' if the lounge has a window.

- No - the statement is not true; for example, the item 'The lounge lighting can be dimmed' would be scored as 'no' if there was no dimmer switch for the room's fixed ceiling lights.

- Not in use - a particular building feature is present, but it is not available for use at the time of the evaluation; for example, the item 'There is a small tea kitchen adjacent to the lounge' would be scored as 'not in use' if the kitchen was not equipped for tea making at the time of the evaluation

- $\mathbf{n} / \mathbf{a}$ - the statement is not applicable; for example, the item 'The reception desk is wheelchair accessible' would be scored ' $n / a$ ' if there was no reception desk.
In an 'ideal' building the answer to each statement would be 'Yes', although it is recognised that in reality there is no such thing as the ideal building.

The 'not in use' response option is primarily for evaluation of existing housing stock, and allows the results page to indicate the extent by which the overall score could be increased if nonfunctioning features were brought back into use.

Although items that are not scored are recorded in the results table as ' $n / a$ ', the ' $n / a$ ' response option enables a person undertaking an evaluation to make it clear that they have deliberately chosen 'not applicable' and have not unintentionally left a question unanswered.

\section{Dependent items}

Similarly, some items are dependent on others and will be scored as ' $n / a$ ', and are shown as greyed out, unless the item on which they depend has been scored as 'Yes'. For example, the item 'The lounge receives sunlight in the morning or afternoon' is dependent on the item 'The lounge has natural light'.

The ' $n / a$ ' response option and the dependent items increase the tool's flexibility and allow it to be used in a wide variety of contexts.

\section{What the tool reveals about a housing scheme or dwelling}

The items in EVOLVE when scored contribute to domain scores representing user needs. There are

\section{Table 1: EXTRACT FROM THE EVOLVE TOOL, SHOWING THE FOUR RESPONSE OPTIONS}

No. ITEM
$\begin{aligned} & \text { The kitchen drawer handles are D-shaped } \\ & 2 \text { The kitchen has an oven }\end{aligned}$
3 There is more than $500 \mathrm{~mm}$ of unbroken worktop each side of the oven
4 in use
Note.
Item 3 is dependent on item 2, and appears greyed-out unless item 2 is scored as 'Yes'. Item 4 is
a generic item, and is scored automatically once the items in the generic item checklist have been
completed.


EVOLVE: a tool for evaluating the design of older people's housing

\section{Table 2: THE DOMAINS}

\section{Universal needs}

Personal realisation The degree to which the building enables residents to engage in their and choice chosen activities and lifestyle Example: There are areas in the garden where residents can grow plants and vegetables

Dignity and privacy The degree to which the building enhances dignity and affords residents privacy

Example: There is a WC which can be accessed without going through a bedroom

Comfort and control The degree to which residents have control over temperature and ventilation

Example: The room or radiator has an individual thermostatic temperature control

Personal care The degree to which the building enables residents to perform activities of personal care, such as washing and bathing

Example: The bathroom has a shaver point

Social support

The degree to which the building enables residents to socialise within inside building the housing scheme

Example: There is space in the lounge for a minimum of four people to sit down within a $3.5 \mathrm{~m}$ diameter without rearranging furniture

Social contact The degree to which the building enables residents to socialise outside outside the housing scheme, through its design and location Example: The scheme is located within a $400 \mathrm{~m}$ travel distance of a public transport terminus such as a bus stop

\section{Support for older age}

Accessibility

The degree to which design features enable residents to move freely around the building without assistance

Example: There is space inside the hallway for a wheelchair turning circle more than $1500 \mathrm{~mm}$

Physical support The degree to which design features enable physically frail residents to have independence

Example: Handrails are provided along travel routes

Sensory support The degree to which the building ameliorates the effects of sensory impairments such as sight loss or hearing difficulties Example: The electric light illuminance is more than 200 lux

Dementia support The degree to which the building supports the needs of residents with dementia

Example: Public areas are indicated by their larger scale and higher ceilings 


\section{Table 2: THE DOMAINS (CONTINUED)}

Health and safety
$\begin{aligned} & \text { The degree to which the building provides a safe environment which } \\ & \text { promotes good health } \\ & \text { Example: The lounge has an alarm call }\end{aligned}$
The degree to which the building provides a secure environment
Example: The scheme front door has an entry phone system
The degree to which the building enables staff to deliver the highest
Standard of care
Example: There is more than $600 \mathrm{~mm}$ round the sides of the washbasin
to allow a carer to be present

13 domains in total, conceptually split into two categories: universal housing needs that apply to people of all ages, and those needs which particularly apply to older people. The domains are defined as shown in Table 2.

Results are displayed in tables and in histograms, with a score for each domain indicating how well a building caters for a particular need. The results do not show an absolute score; rather, a profile is created of the scheme or dwelling, showing its strengths and weakness. The advantage of this approach is that it is possible to compare large schemes, such as retirement villages, with smaller schemes. However, it also means that the number of communal facilities in a scheme is not reflected in the domain scores, although the available facilities are recorded in the accommodation schedule.

The results of an evaluation can be displayed at a range of scales, from broad to detailed. Results can be obtained for the whole scheme, for part of a scheme (such as for an individual apartment or bungalow, or for all the communal facilities) or for individual rooms. As the tool is essentially a checklist, it is also useful for recording information at item level.

The EVOLVE tool is available from the Housing LIN website: www.dhcarenetworks.org.uk/.

\section{Address for correspondence}

Alan Lewis

School of Architecture

University of Sheffield

Crookesmoor Building

Conduit Road

Sheffield S10 1FL

UK

a.lewis@sheffield.ac.uk

\section{References}

CLG (2008) Lifetime Homes, Lifetime neighbourhoods: A national strategy for housing in an ageing society. London: Communities and Local Government.

Evans G, Kantrowitz E \& Eshelman P (2002) Housing quality and psychological well-being among the elderly population. The Journals of Gerontology: Series B, Psychological Sciences and Social Sciences 57 (4) 381-3.

Parker C, Barnes S, McKee K, Torrington J \& Tregenza P (2004) Quality of life and building design in residential and nursing homes for older people. Ageing and Society 24 (6) 941-62.

Thomson H, Petticrew M \& Morrison D (2001) Health effects of housing improvement: systematic review of intervention studies. British Medical Journal 323 (7306) 187-90.

Wahl H-W, Schilling O, Oswald F \& Iwarsson S (2009) The home environment and quality of life-related outcomes in advanced old age: findings of the ENABLEAGE project. European Journal of Ageing 6 (2) 101-11. 